\title{
Local Heat Transfer and Pressure Drop in a Rotating Two-pass Ribbed Rectangular Channel
}

\author{
SHOU-SHING HSIEH* and HSIU-CHENG LIAO \\ Department of Mechanical Engineering, National Sun Yat-Sen University, Kaohsiung, Taiwan 80424, Republic of China
}

(Received 5 May 2000; In final form 27 May 2000)

\begin{abstract}
The influences of rotation and uneven heating condition as well as passage aspect ratio on the local heat transfer coefficient and pressure drop in a rotating, two pass ribroughened (rib height $e / D_{H} \approx 0.27$; rib pitch $p / e=8$ ) rectangular channel with a crosssection aspect ratio of 3 was studied for Reynolds numbers from 5000 to 25,000 and rotation numbers from 0 to 0.24 . Regionally averaged Nusselt number variations along the duct have been determined over the trailing and leading surfaces for two pass straight channels and U-bend region. Implementing with the data from Hsieh and Liu (1996) for $A R=1$ and 1.5 with $p / e=5$ and $e / D_{H}=0.17$ and 0.20 , passage aspect ratio effect was further examined. Furthermore, data for $180^{\circ}$ U-bend region with ribroughened turbulator on heat transfer were also measured. It was found that a complicated three-dimensional accelerated flow and secondary flow in this U-bend region caused higher heat transfer on both leading/trailing walls. Enhancement performance ratios are also presented and discussed. Results again indicate a slight decrease in heat transfer coefficient for an increase in passage aspect ratio as compared to those of previous studies.
\end{abstract}

Keywords: Heat transfer; Pressure drop; 2 pass rotating channel; Rib turbulator; $180^{\circ}$ U-bend

\section{INTRODUCTION}

Owing to the increase in the turbine inlet temperature of gas turbine engines, there is an urgent need today to obtain a higher efficiency in the engines of aircraft, ships and many other industrial applications. Parallel with the evolution of metal working at high temperatures, several methods of cooling rotor blades have been tried and developed. Cooled blades are widely used in modern engines. Radial channel cooling is a commonly used method. These channels are often designed with two artificially roughened and two smooth walls, and the designer must know the heat transfer coefficient on each of the walls in order to predict the turbine airfoil's life correctly. Naturally, it is also necessary to know the pressure loss for such a channel. Highly sophisticated cooling

\footnotetext{
*Corresponding author. Fax: (07)5254009, e-mail: sshsieh@mail.nsysu.edu.tw
} 
techniques such as augmented internal convective cooling have been employed for turbine blades in order to maintain acceptable safety requirements under extreme operating conditions. However, it is important to understand the effect of blade rotation on local heat transfer coefficient distribution inside the serpentine coolant passages (Yang et al., 1992) and the surface heating conditions.

As the increase in heat transfer rate is accompanied by a friction factor increase, the preferred roughness geometry will yield the desired heat transfer augmentation with a minimum friction factor. The internal passages can be approximately modeled as square or rectangular channels with two oppositely rib-roughened walls. The heat transfer and friction characteristics in these channels are different from those of circular tubes, parallel plates, or annuli (Hong and Hsieh, 1993; Hsieh and Hong, 1995).

Rotation of turbine blade cooling passages give rise to Coriolis and buoyancy forces that can significantly alter the local heat transfer in the inward coolant passage from the development of cross-stream (Coriolis), as well as radial (buoyant) secondary flows (see, for example, Wagner et al., 1992). Buoyancy forces in gas turbine blades are substantial because of high rotational speeds and coolant temperature gradients. Therefore, a better understanding of Coriolis and buoyancy effects and the capability to predict the heat transfer response to these effects will allow the turbine blade designer to achieve optimum cooling configurations that utilize less flow and, consequently, reduce the compressor power as well as thermal stresses in the airfoil. In addition, under realistic conditions, the blade surface is subjected to different or uneven heat flux or temperature on the leading or trailing surface of the serpentine square channels. Furthermore, it is shown (Parsons et al., 1994; Hsieh and Liu, 1996) that the uneven wall heat flux/temperature creates local buoyancy forces that would change the effect of the rotation, which results in changes in the local heat transfer coefficients on the leading/trailing surface due to the uneven heat flux especially for multipass flow channels (Parsons et al., 1994; Zhang et al., 1995). The results presented in this paper are aimed at studying the local heat transfer and pressure drop in a rotating two-pass ribbed rectangular channel. The channel aspect ratio effect on heat transfer and pressure drop data were presented and discussed.

The study presents friction and heat transfer measurements from both the straight channels (first and second pass) and the U-bend region with rib-roughened surfaces in a rotating rectangular channels of aspect ratio 3. Measurements were obtained for pressure drop and local temperature distribution. Also, the geometric parameters, such as $A R, p / e$, and $e / D_{H}$ of this investigation are different from those of Hsieh and Liu (1996).

\section{EXPERIMENTAL APPARATUS AND PROCEDURE}

The present test facility is similar to that used by Hsieh and Liu (1996) with a slight modification in rib placement design (including U-bend region) and flow passage. The facility comprised a heat section, a blower, a motor, a heat source, two slip ring assemblies, and a datalogger. The test section consisted of a ribbed two-pass serpentine flow channel with $1.0 \times 3.0 \mathrm{~cm}$ rectangular cross sectional channel, mounted on a shaft supported by two bearings. The rotating shaft speed is measured by a digital photo tachometer. The air used for the test fluid was blown through the test section via a rotating seal assembly in the radially outward direction with a $2 \mathrm{~kW}$ centrifugal blower. A 34.0-mm-dia. pipe, equipped with a 17.0-mm-dia. orifice plate $\left(C_{0}=0.66\right)$, was used to measure mass flow rate. After passing through the orifice plate, the air enters the plenum cylinder in the hollow shaft and it flows through the straightener fixed in the interior of the shaft to eliminate the initial swirl before entering the rectangular duct. The Reynolds numbers based on the channel hydraulic diameter $\left(D_{H}\right)$ ranged from 5,000 to 25,000 . 
To determine the locally averaged streamwise heat transfer coefficients in the channel along the flow direction, the two-pass test flow channel is divided into 9 sheet copper sections (Hsieh and Liu, 1996) (see Fig. 1 for details). Each copper section is composed of two copper plates $(32 \times 10 \times 1 \mathrm{~mm}$ each) with two other sides made of plexiglass, with an inner cross section of $10 \mathrm{~mm}$ by $30 \mathrm{~mm}$. The Teflon strips are machined along periphery contact surface between copper section for insulation to prevent possible heat conduction. The ratio of channel length to hydraulic diameter $\left(L / D_{H}\right)$ is 40.4 , which gives that ratio for each straight channel length of 19.2 and U-bend of 2, respectively. The ratio of the mean rotating arm radius to the channel hydraulic diameter is 20 . The rib is oppositely positioned on two walls of the rectangular test channel. However, the rib placed in the U-bend was different and also indicated in Figure 2. The rib height-to-hydraulic diameter ratio $\left(e / D_{H}\right)$ is 0.27 . The rib pitch-to-height ratio $(p / e)$ is 8 and the angle between the rib and coolant flow direction equals $90^{\circ}$. The channel aspect ratio is 3 . The ribbed leading and trailing surface were made by gluing plexiglass ribs (each $10 \times 4 \times 4 \mathrm{~mm}$ ) which are different from those in real engines with low thermoconductivity, in between two copper plates to the plexiglass rectangular channel on opposite walls in a required manner. Each wall has its own thin film $(0.1 \mathrm{~mm})$ resistance heater powered by a variac transformer for controlled heat fluxes. The power to each element was adjusted to obtain an uneven heating condition on each leading/and trailing walls. The smooth side walls are insulated from the leading and trailing walls to minimize heat losses to the environment. The entire heated section is insulated by $2-\mathrm{mm}$-thick styrofoam. The local wall temperature of the test section is measured by 21 AWG40 copper-constantan thermocouples

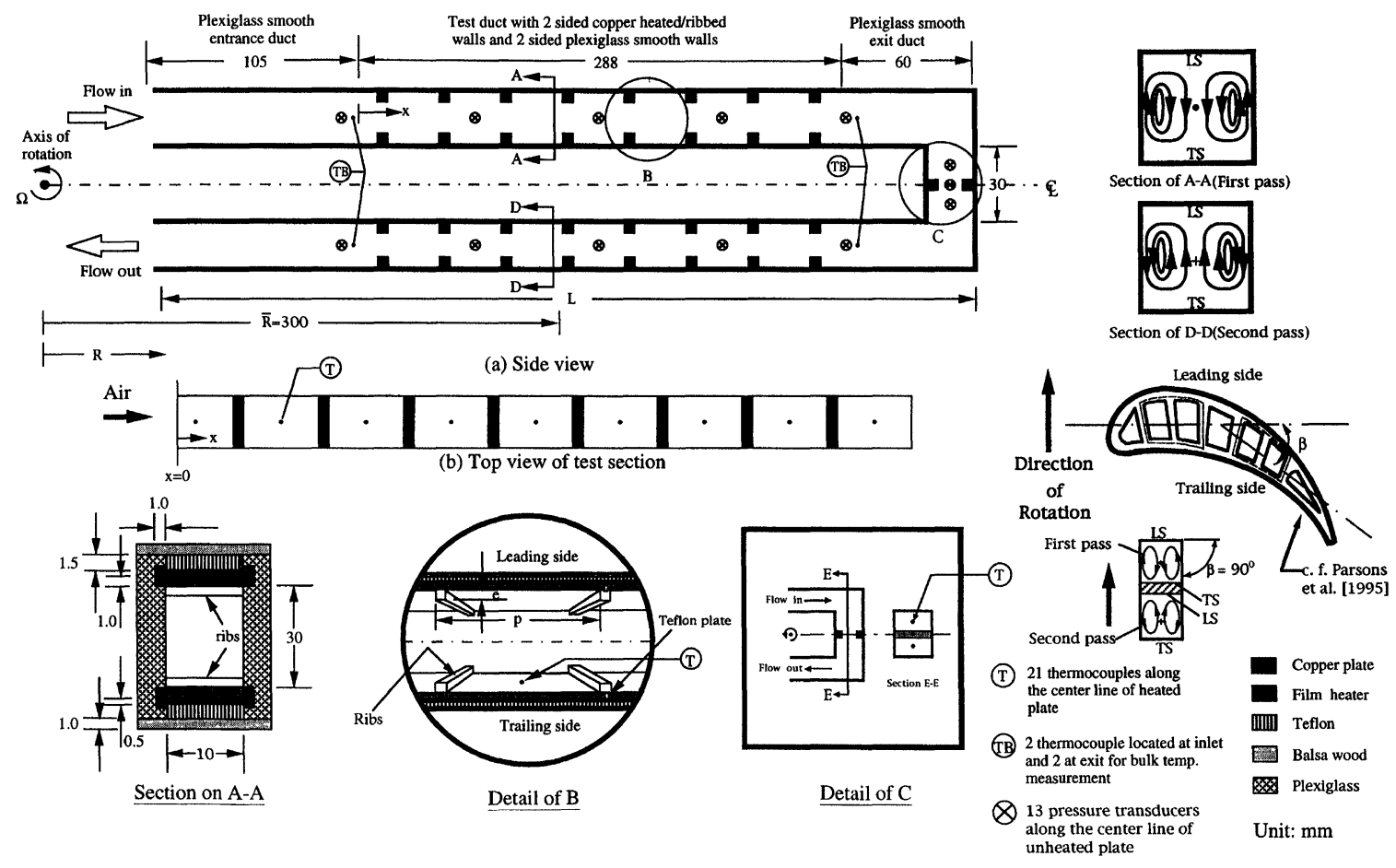

FIGURE 1 Detailed thermocouple and pressure drop measurement of the test section $(A R=3)$. 


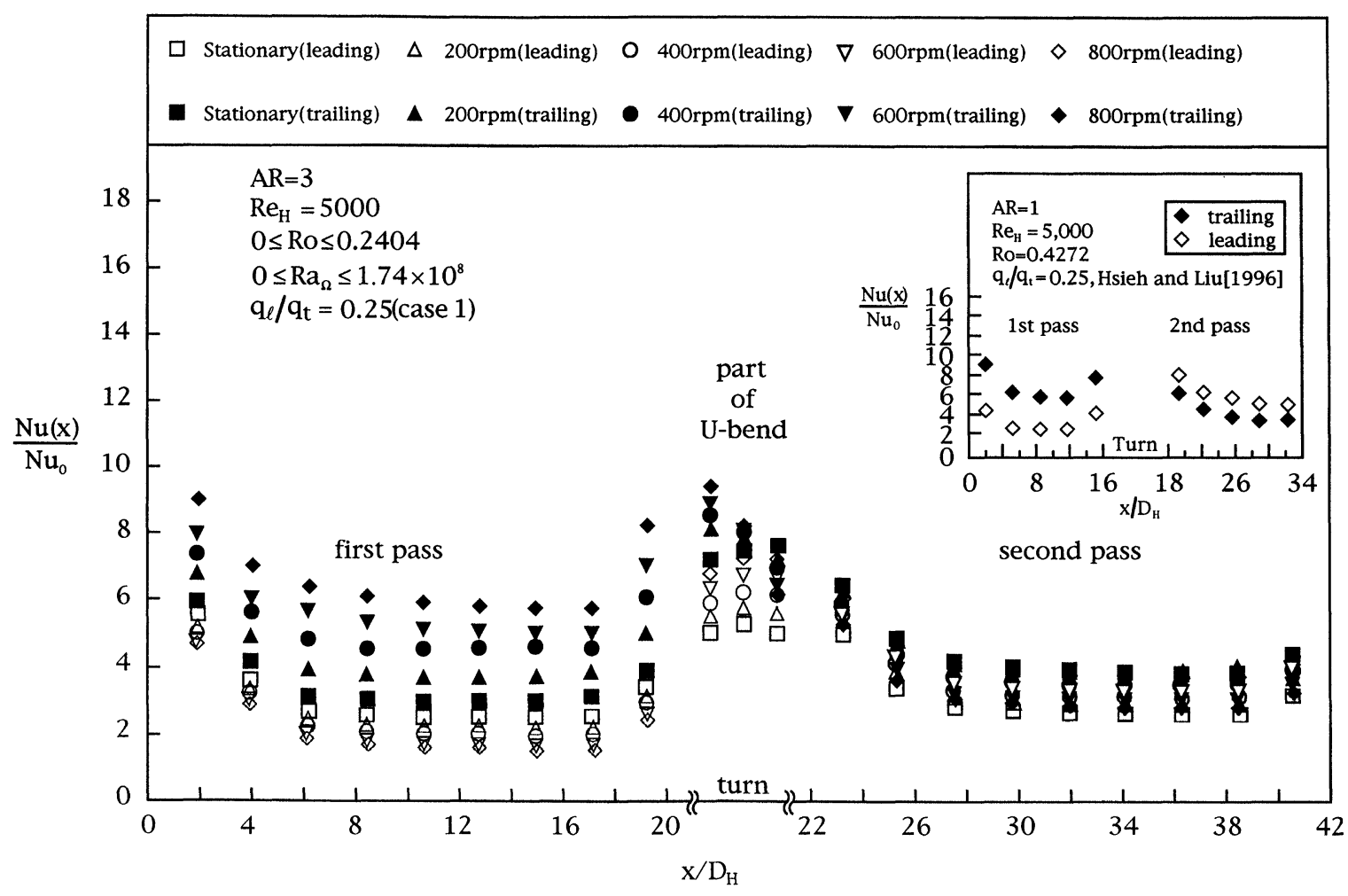

FIGURE 2 Effect of rotation on Nusselt number ratio $\left(q_{\ell} / q_{t}=0.25\right)$.

TABLE I Geometries and operating condition in the channel

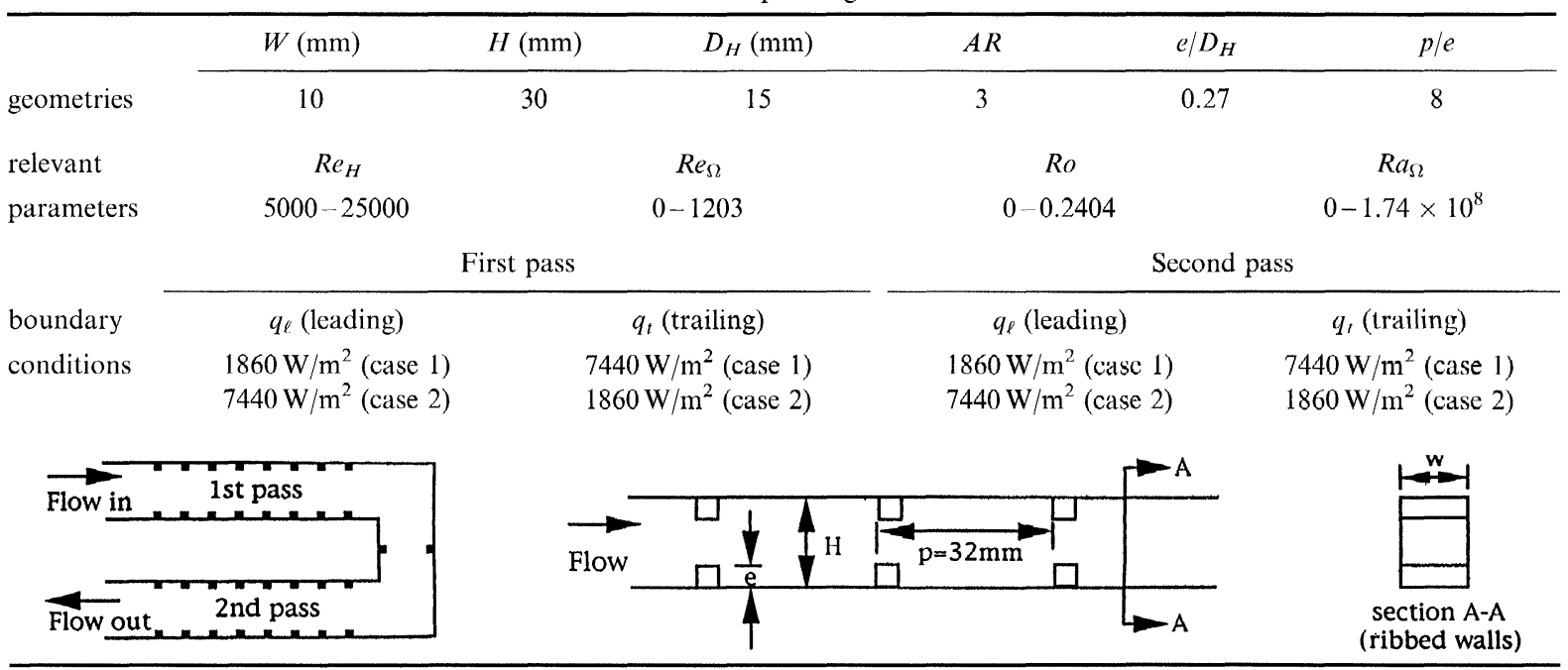

distributed along the downstream flow direction and around the perimeter of copper test section. Two more thermocouples measure the inlet and outlet bulk air temperature. There is an unheated plexiglass entrance duct that has the same cross section and length $\left(x / D_{H} \approx 7\right)$ shorter than that 
of the test duct. A Schlumberger Solartron 3530 datalogger was interfaced with an IBM PC/AT for data acquisition.

Experimental data were taken on the leading and trailing surfaces of the test section in both rotating and stationary channels. Rotational speeds of 200, 400, 600 and $800 \mathrm{rpm}$ were set in the counterclockwise(forward) direction. The test channel geometry and operating conditions are listed in Table I.

\section{DATA REDUCTION AND UNCERTAINTY ANALYSIS}

The friction factor was calculated from the pressure drop across the flow channel and the air dynamic pressure as $\Delta P /\left(0.5 \rho U^{2}\right)$. The results were normalized by the corresponding local friction factor for smooth circular tubes. One of the main goals is to analyze the local (regionally averaged) heat transfer phenomenon on both leading and trailing surfaces. The heat transfer quantitative analysis is done by Nusselt number. In order to determine the local (regionally averaged) Nusselt number, it is necessary to obtain the axial variation of heat flux from the passage wall and the difference between the wall temperature and the bulk mean temperature of the coolant. To get the local Nusselt number, one uses the equation $h(x)=$ $Q_{\text {net }} /\left[A\left(T_{w}-T_{b x}\right)\right]$, where $h(x)$ is the local heat transfer coefficient, $\left(Q_{\text {net }} / A\right)$ is the local heat transfer rate per unit area from the wall to the cooling air, $T_{w}$ is the local wall temperature of the plate, and $T_{b x}$ is the bulk mean air temperature. The surface area is the projection area which does not include the surface of the rib. The electrical power generated from the heater is determined from the measured heater resistance and voltage on each wall of the test duct. The effect of axial wall conduction between copper sections on the local net heat transfer rate is less than 3.06 percent, which is also included in the data analysis.

The value of $T_{b x}$ is obtained by energy balance, and defined as $T_{b x}=T_{0}+\sum Q_{\text {net }} /\left(G C_{p}\right)$, where $\sum Q_{\text {net }}$ is the summation of net heat given to the air from the entrance (about $27^{\circ} \mathrm{C}$ ) to a specified positions along the downstream distance. The heat loss is the amount of heat conducted into the test section and test stand but not converted directly into the air. Tests for heat loss are performed at the same rotating speed, without coolant flow. All input power levels are used to determine heat losses as functions of temperature differences between the copper plate and the surroundings. It is found that the average ratio of heat loss power-to-total power input is from 0.10 to 0.22 at $R e_{H}=5000$ as rotational speed increase from 0 to $800 \mathrm{rpm}$. The heat conduction between adjacent copper plates was less than $1.5 \%$ of the total power input to the copper plates. The local Nusselt number was calculated by $N u=h(x) D_{H} / K_{f}$ where $K_{f}$ is the thermal conductivity of air. The local Nusselt number, normalized by the Nusselt number for a stationary fully developed turbulent flow in a smooth circular tube, is correlated by Dittus-Boelter/McAdams (Rohsenow and Choi, 1961) as:

$$
N u / N u_{0}=\left(h D_{H} / K_{f}\right) /\left[0.023 R e^{0.8} \operatorname{Pr}^{0.4}\right]
$$

with $\operatorname{Pr}=0.7$. The uncertainty in temperature measurement is estimated to be $\pm 0.5^{\circ} \mathrm{C}$. The maximum nondimensional uncertainty intervals for the variables concerned in this study are estimated and given in Table II.

TABLE II Typical nondimensional interval for the relevant variables

\begin{tabular}{lll}
\hline \multicolumn{1}{c}{ Variables } & & Uncertainty \\
\hline Specific heat of air & $C_{p}$ & $\pm 3 \%$ \\
Hydraulic diameter of flow pass & $D_{H}$ & $\pm 5.4 \%$ \\
Air flow rate & $G$ & $\pm 5.3 \%$ \\
Thermal conductivity of air & $k$ & $\pm 0.75 \%$ \\
Temperature & $T$ & $\pm 0.5^{\circ} \mathrm{C}$ \\
Rotational speed & $\Omega$ & $\pm 1 \%$ \\
Reynolds number & $R e_{H}$ & $\pm 7.9 \%$ \\
Rotational Reynolds number & $R e_{\Omega}$ & $\pm 12.4 \%$ \\
Local Nusselt number & $N u$ & $\pm 16.8 \%$ \\
Pressure drop & $\Delta P$ & $\pm 1.5 \%$ \\
Friction factor & $f$ & $\pm 12.1 \%$ \\
\hline
\end{tabular}




\section{RESULTS AND DISCUSSION}

The convective heat transfer performance inside of a rotating two-pass roughened channel is governed by the ratio of the rotating mean radius to channel hydraulic diameter, Reynolds number, Prandtl number, rotation number, rotational Rayleigh number, channel aspect ratio, wall to fluid density difference ratio, flow direction (radial outward flow/or radial inward flow), and rib geometry and size. For the present study, both $\bar{R} / D_{H}=20$ (4080 in real engines) and $\operatorname{Pr}=0.7$ are fixed and rib geometry and size are specified. The fluid flow rate, heating rate ratio $\left(q_{\ell} / q_{t}=1\right.$ and 4$)$, and rotational speed $(\Omega=0,200,400,600,800 \mathrm{rpm})$ of the rotor, were varied to produce various values of $R e_{H}, R o$, and $(\Delta \rho / \rho)_{i}$. Besides, incorporating with the data from Hsieh and Liu (1996) for $A R=1$ and 1.5, measurements of an aspect ratio of 3 was also conducted to further examine the effect of aspect ratio.

\section{Heat Transfer Results (In First/Second Channel and U-bend Region)}

\section{Effect of Rotation and Uneven Heating Condition}

The local (regionally averaged) heat transfer results are presented as the axial distributions of a normalized Nusselt number ratio. Figures 2 and 3 show the effect of rotation for case 1 wall heating condition on the local Nusselt number ratio $(\mathrm{Nu} /$ $N u_{0}$ ) on the leading and trailing walls for first/ second channel and U-bend region. In Figure 2 for $R o=0$ and 0.24 at $R e_{H}=5000$, local Nusselt number ratios for the ribbed leading and trailing walls are fairly uniform similar to those of Hsieh and Liu (1996) throughout the two-pass test channel. However, the values of the present study seem lower than those of Hsieh and Liu (1996) due to different $e / D_{H}$ and $p / e$ as well as $A R$. Despite this, for the U-bend region, the local $N u$ values drastically increase first and, then decrease to a certain value as the flow approaches downstream.
Generally, the stationary heat transfer ratio for the present case is about $2.2-2.8$ in the most portion of the channels.

In the first outflow pass $\left(0<x / D_{H} \leq 20\right)$, the local Nusselt number ratios for the trailing wall increase while the Nusselt number ratios for the leading wall decrease relative to their corresponding nonrotating value as $R o$ increases from 0 to 0.24 . The reverse holds in the inflow pass $(23 \leq$ $x / D_{H}<42$ ). This is because rotation induces the Coriolis forces that produce secondary crossstream flows and it makes the first outflow pass trailing and second inflow pass leading boundary layers become thinner. Simultaneously, it also thickens the first pass leading and the second pass trailing boundary layers. Due to such thinning or thickening of the boundary layers, the heat transfer coefficients for the first pass trailing and second pass leading walls are higher with rotation than those without.

On the contrary, the heat transfer coefficients for first pass leading and second pass trailing walls are lower with rotation than those without. The decrease in heat transfer at the leading(first pass)/ trailing(second pass) surface due to rotation can be explained by the reverse of the reasons stated before. This behavior is reduced as $R o$ decreases. The above mentioned findings agree well with those reported by Hsieh and Liu (1996) which are also shown in Figures 2 and 3 for the corresponding cases (actually, $p / e$ and $e / D_{H}$ are little bit different) except for the U-bend region and the different $R o$. Moreover, the present results exhibit a local peak value (about 10 at $\operatorname{Re}_{H}=5000$ ) in the vicinity of the U-bend. Namely, followed by an entrance decrease; decreasing $\mathrm{Nu} / \mathrm{Nu} u_{0}$ with increasing $x / D_{H}$ starting from upstream $90^{\circ}$ sharp turn until reaching the downstream $90^{\circ}$ sharp turn. Other than the U-bend region the general trend of Figures 2-4 shows a similar behavior as that of Hsieh and Liu (1996). Further inspection of Figures 2 and 3 indicates the effect of flow rate on local heat transfer performance. It is found that an increase in $R e_{H}$ reduces the magnitude but preserves the shape of the $N u / N u_{0} \quad v s . x / D_{H}$ as 


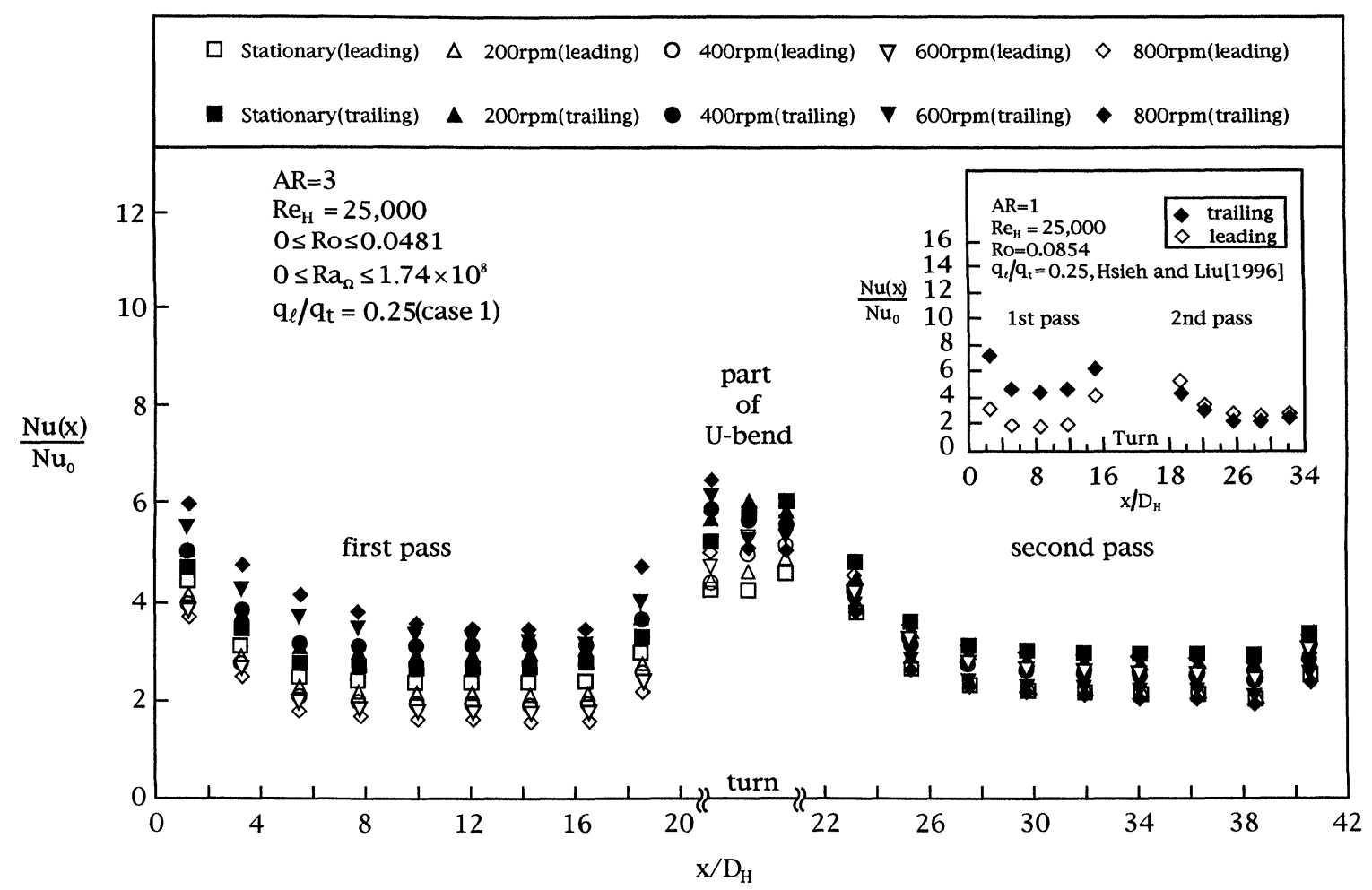

FIGURE 3 Effect of rotation on Nusselt number ratio $\left(q_{\ell} / q_{t}=0.25\right)$.

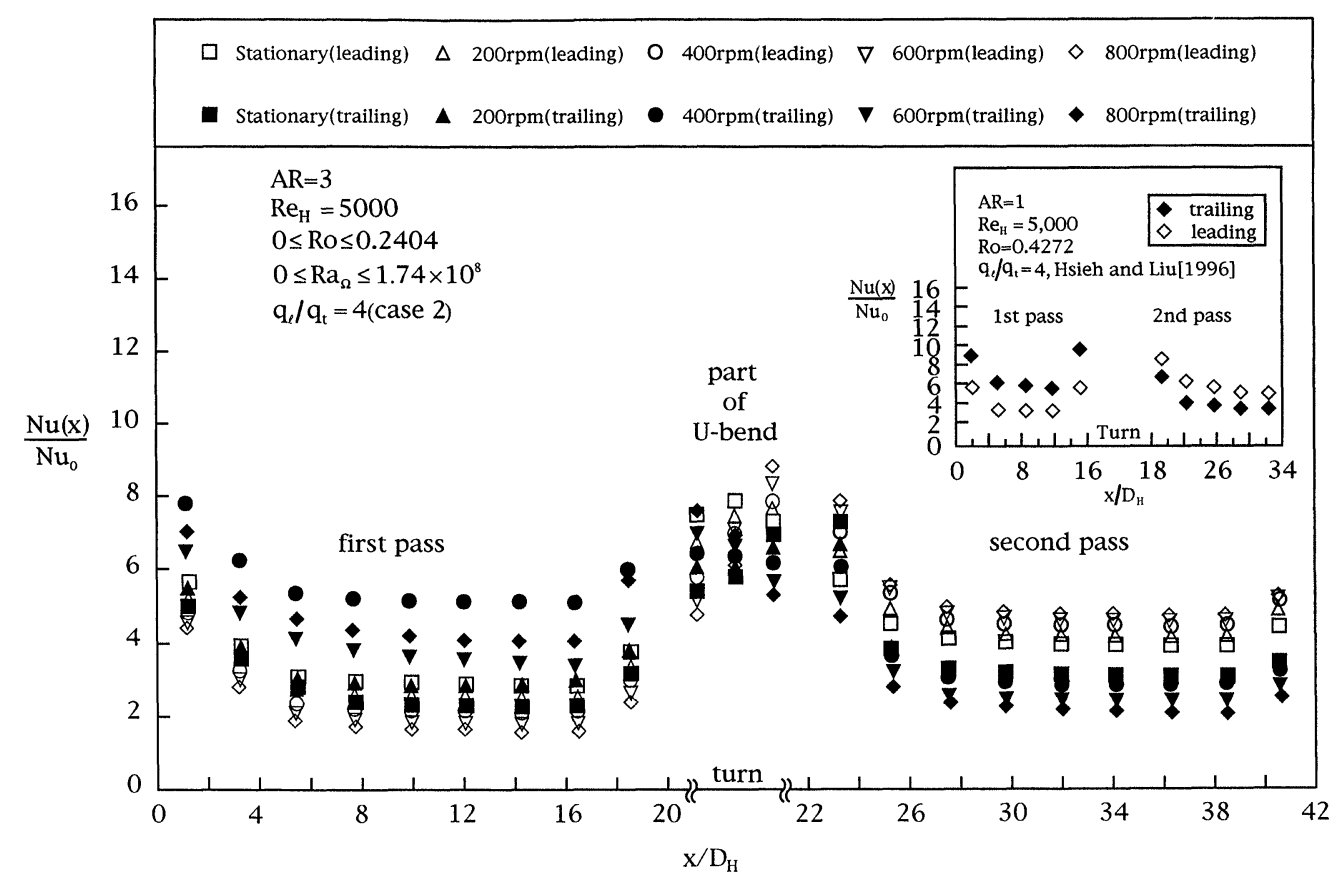

FIGURE 4 Effect of rotation on Nusselt number ratio $\left(q_{\ell} / q_{t}=4\right)$. 


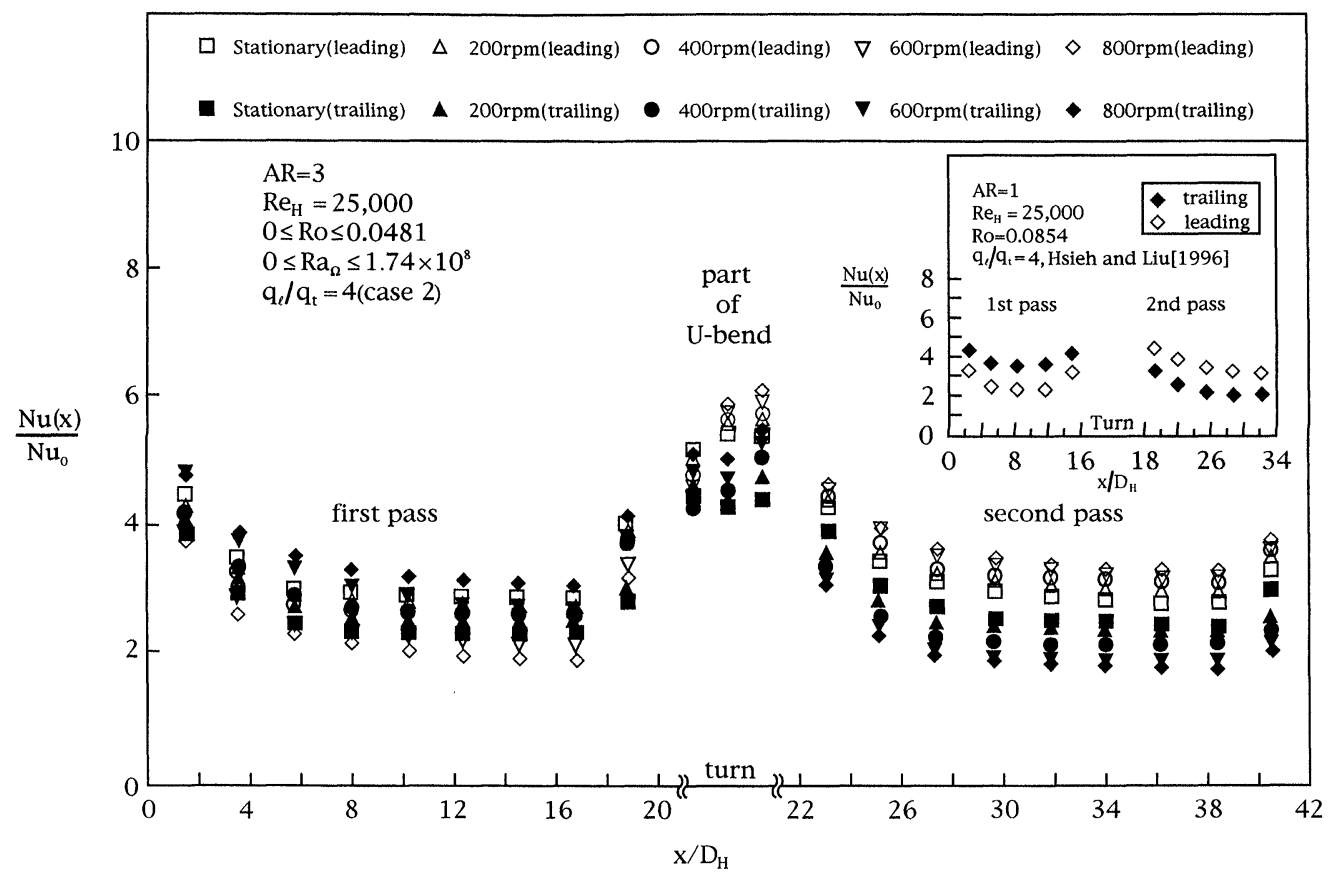

FIGURE 5 Effect of rotation on Nusselt number ratio $\left(q_{\ell} / q_{t}=4\right)$.

expected. Figures 4 and 5 show the same results except for $q_{\ell} / q_{t}=4$. It indicates that the larger uneven heat flux ratio like $q_{\ell} / q_{t}=4$ is the lower heat transfer enhancement exhibits. This also agreed well with those reported by Hsieh and Liu (1996). The reason can be seen from Hsieh and Liu (1996). However, the magnitude of the present $\mathrm{Nu}$ / $N u_{0}$ appears a little bit lower than those of Hsieh and Liu (1996) as also evidenced by Figures 4 and 5 due to a higher aspect ratio of the present study which will be discussed later.

\section{Effect of Centrifugal Buoyancy, Uneven Heating Conditions and Aspect Ratios}

When tests were conducted at a fixed Reynolds number $\left(R e_{\Omega}\right)$ and rotational speed, but with varying levels of wall-to-fluid bulk temperature difference, evidence of a buoyant interaction was noted. Figure 6 plotted the piecewise (e.g., first/ second channel, and U-bend) averaged $\overline{N u}$ against the $R a_{\Omega} / R e_{H}^{2}$ for $A R=3$. It is found that the $\overline{N u}$ on leading surface increases with $R o$. The reason is that as the buoyancy increases the leading side heat transfer, the Coriolis-induced secondary flow entrains this "hotter" fluid and deposits it on the trailing edge resulting in an accompanied reduction in heat transfer with an increase $R o$ due to a mild temperature gradient. Moreover, it is also found, that an increase in $\overline{N u}$ with an increase in $R a_{\Omega} / R e_{H}^{2}$ occurred on both leading and trailing surfaces in the first/and second channel for both $R e_{H}=5000$ and 25000. However, such a centrifugal buoyancy dependence is much different on the leading/and trailing surface in the U-bend region. It is found on the leading surface the centrifugal buoyancy seems weaker and can not observed. This is expected due to the present centrifugal force. This situation becomes much clearer as $R o$ increases. The data from Hsieh and Liu (1996) for $A R=1$ are also included for comparison. The present results are much higher than that of Hsieh and Liu (1996) because of a much higher $A R$ of the present study causing a higher centrifugal 


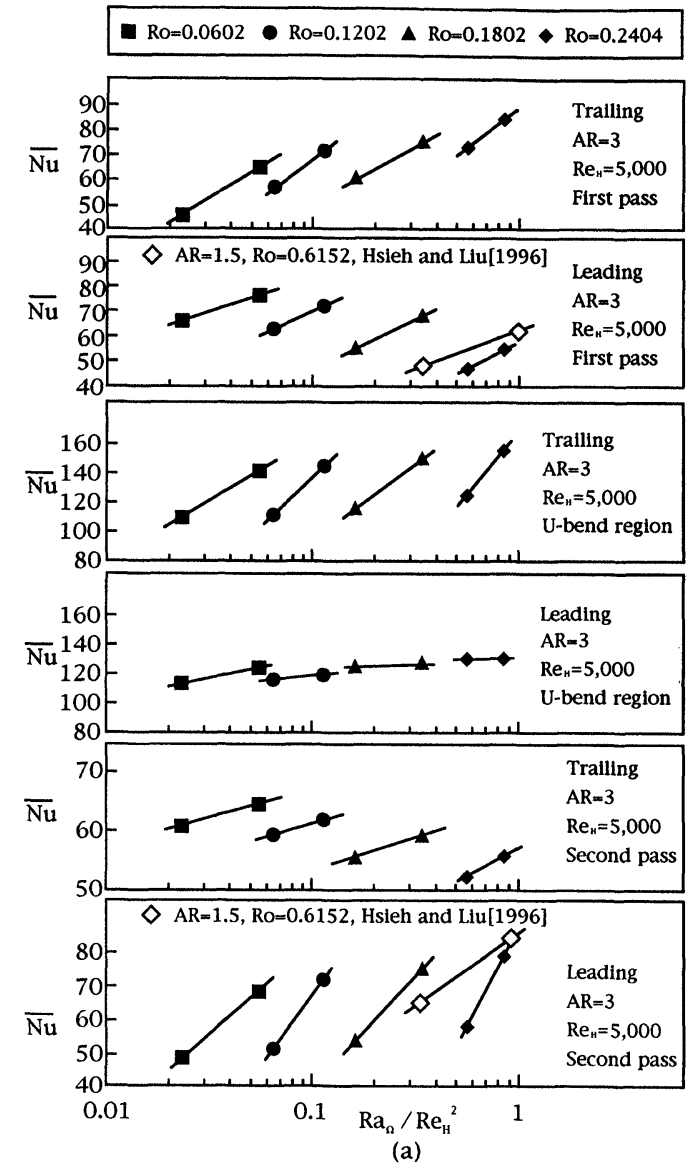

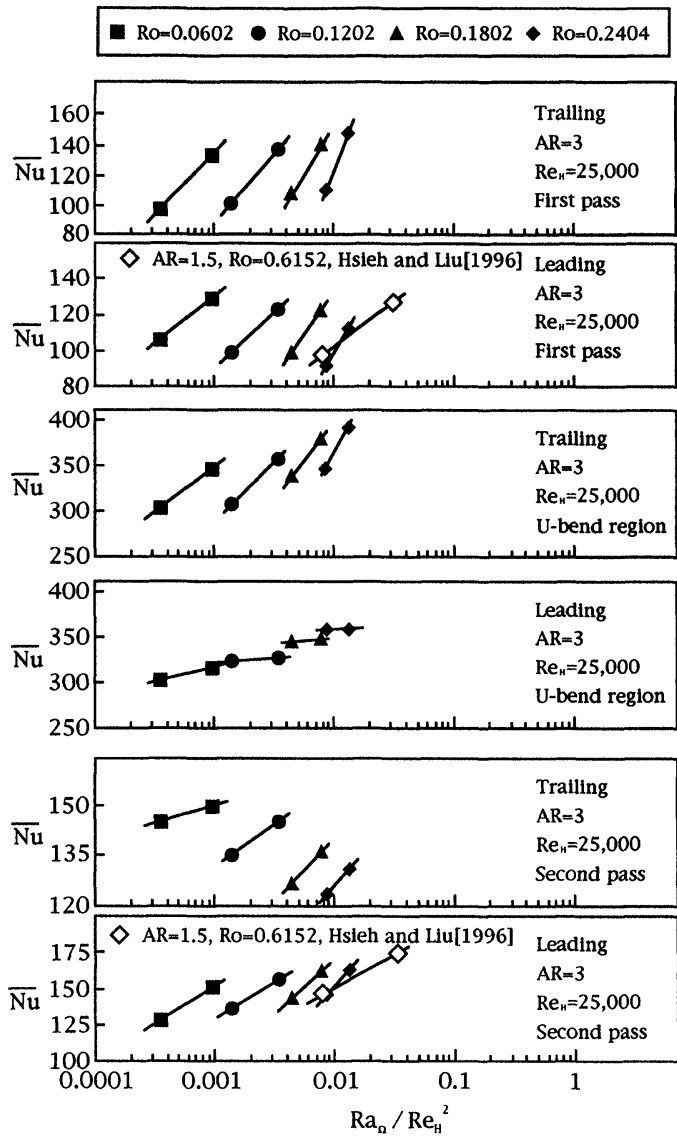

(b)

FIGURE 6 Centrifugal buoyancy effect on Nusselt number $(A R=3)$.

buoyancy. This behavior may be due to the relative space between roughened walls. Consequently, it reduces the throughflow velocity, the velocity gradients, turbulent kinetic energy, and, hence, convection is weaker. Due to the different $p / e$ and $e / D_{H}$, the comparison may be somewhat in appropriate. But, nevertheless, the aspect ratio effect in any sense is significant.

\section{Pressure Drop Results}

\section{and Enhancement Factor $(\boldsymbol{\eta})$}

As stated earlier, pressure drop measurements were conducted with $(R o=0.06$ and 0.18$) /$ without rotation for both smooth and ribbed U-bend. The smooth stationary channel corresponding value was used as a reference for vertical ordinate. Figure 7 shows variation in static pressure along the top wall of the channel and presents the results for ribbed channel with smooth/and ribbed Ubend at $R e_{H}=5000$ for adiabatic and diabatic cases (three cases were considered). The results clearly indicate that the isothermal friction factor is the least followed by a low $\left(q_{\ell}=q_{t}=\right.$ $\left.1860 \mathrm{~W} / \mathrm{cm}^{2}\right)$, a moderate $\left(q_{\ell}=1860 \mathrm{~W} / \mathrm{cm}^{2}\right.$ and $\left.q_{t}=7440 \mathrm{~W} / \mathrm{cm}^{2}\right)$ and a high $\left(q_{\ell}=q_{t}=7440 \mathrm{~W} /\right.$ $\mathrm{cm}^{2}$ ) heat flux level. Generally, the streamwise $f /$ $f_{0}$ distribution for the straight channels with smooth/and ribbed U-bend is nearly constant in the first channel for all of the cases considered with a 

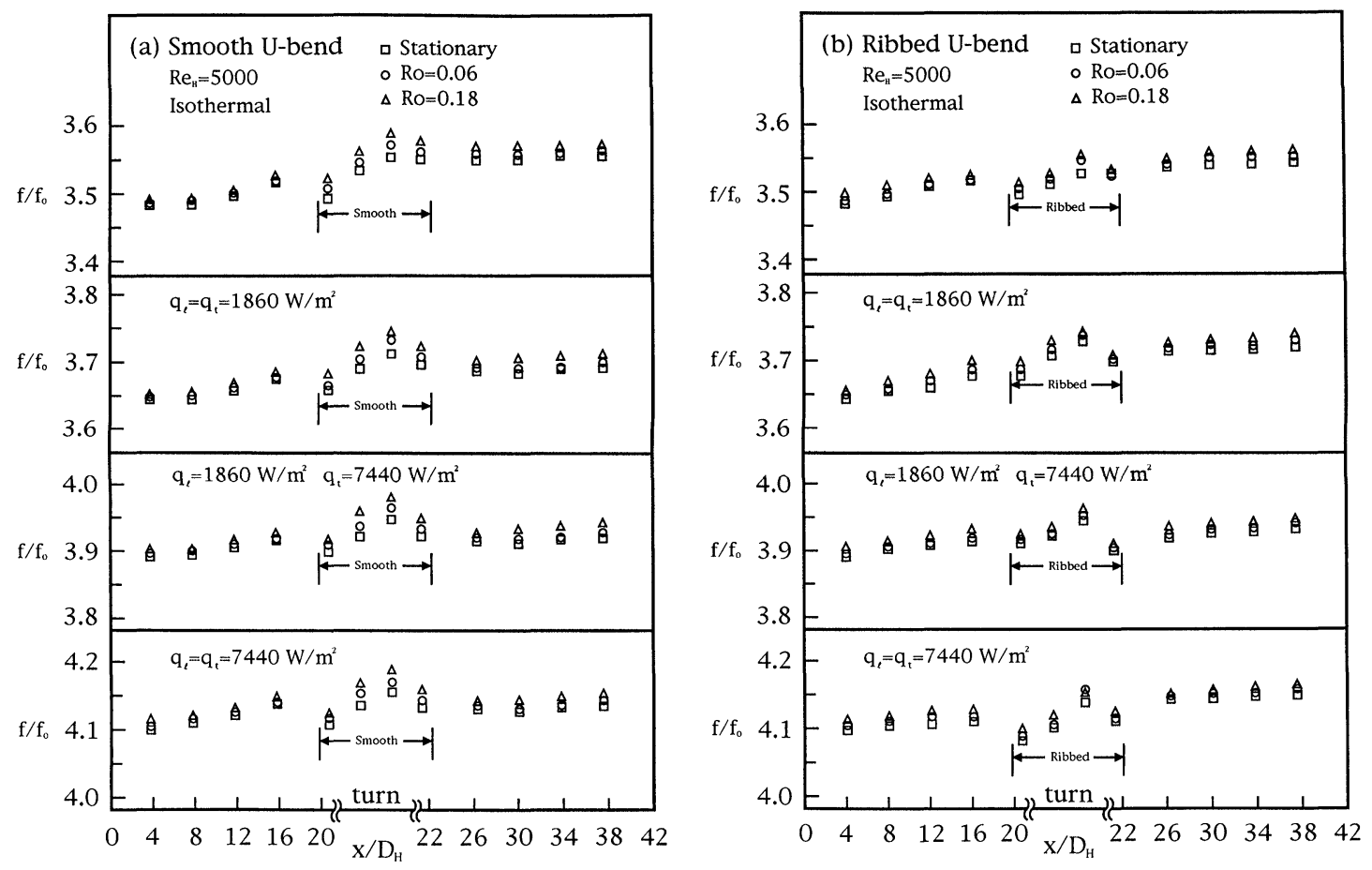

FIGURE 7 Pressure drop variation along the downstream.

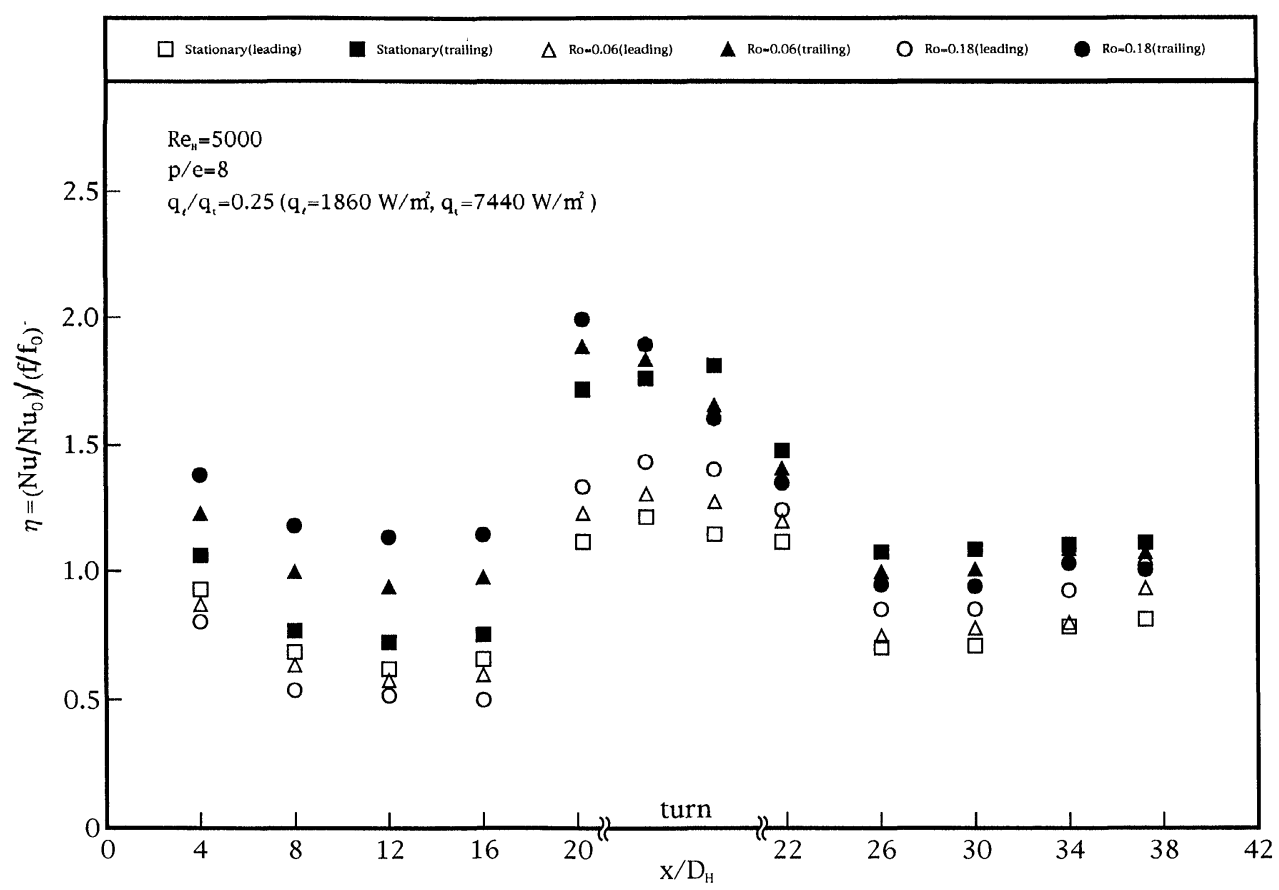

FIGURE 8 Heat transfer performance along the downstream. 
drop right at the inlet of the U-bend followed by a recovery within the U-bend to $x / D_{H} \cong 22$ and then slightly decrease to a constant value for each case eventually at $x / D_{H}=38$ in the second channel as shown in Figures 7(a) and (b). The data also indicate that in both the first and second straight channels, the frictional pressure drops are not significantly affected by rotation. However, the friction factor due to the U-bend influence can be clearly noted especially for the smooth U-bend as shown in Figure 7(a). This is perhaps because the flow separation due to the up/down stream sharp corners occurs at right after (before) the inlet (exit) of the U-bend. Such a behavior becomes a little bit modest due to the rib effect for the ribbed U-bend as shown in Figure 7(b). The values of $f / f_{0}$ for both smooth/and ribbed U-bends seem the same. Maximum $f / f_{0}$ was also found within the both (i.e., smooth/and ribbed) U-bend region. Furthermore, as mentioned earlier, the heat flux level does have something to do with $f / f_{0}$ which is the same as Figure 7 shows. For all the cases under study, the value at each position along the channels increases as $R o$ increases. Finally, the $f / f_{0}$ conventional enhancement performance ratio $(\eta)$, defined as $\left(N u / N u_{0}\right) /\left(f / f_{0}\right)$ distribution with $\eta$ from 0.5 to 2 for all the cases under study along the streamwise distance was presented in Figure 8 . The results show that several values of $\eta$ (for most trailing surfaces) are bigger than 1 and in fact, at several locations within the U-bend regions $\eta$, can be up to 2. The trailing surface has a higher $\eta$ as compared to the corresponding leading surface.

\section{CONCLUSION}

The influences of uneven wall temperature and channel aspect ratio on the local heat transfer coefficients as well as friction factor in a rotating, two-pass rectangular channel with ribbed walls on both straight channels and U-bend region were studied for $R o$ from 0 to 0.24 by varying the Reynolds number from 5000 to 25000 . The main findings are:
(1) The streamwise distribution of heat transfer coefficients in the first channel with rotation is similar to that of developing flow in the entrance region of a passage without rotation and is again in good agreement with previous studies (Han et al., 1993 and Hsieh and Liu, 1996).

(2) The distribution of $N u$ over the most U-bend region was firstly obtained with a sharp increase right after upstream sharp turn and then drop to a certain value at downstream sharp turn.

(3) Channel aspect ratio effect was further examined by incorporating the data from Hsieh and Liu (1996) with the present results. It reconfirms the previous finding of Hsieh and Liu (1996) that an aspect ratio increase would result in a decrease in averaged heat transfer in both straight channels (i.e., first/second pass).

(4) Pressure drop measurements strongly reveals that with the ribbed U-bend the streamwise distribution of $f / f_{0}$ would be lower than that channel with smooth U-bend.

(5) Enhancement performance ratio $(\eta)$ distribution along the streamwise distance was presented by using the conventional ratio of $\left(N u / N u_{0}\right) /\left(f / f_{0}\right)$. It is found that $\eta$ can be up to 2 right after the upstream sharp turn of the U-bend.

\section{NOMENCLATURE}

$A \quad$ projected area

$A R \quad$ channel aspect ratio, $H / W$

$C_{0} \quad$ discharge coefficient

$C_{p} \quad$ specific heat at constant pressure

$D_{H} \quad$ hydraulic diameter $2 \mathrm{WH} /(\mathrm{W}+\mathrm{H})$

e rib height

$f \quad$ local friction factor, $\Delta P /\left(0.5 \rho U^{2}\right)$

$G \quad$ mass flow rate

$g \quad$ gravity

$H \quad$ height of channel

$h \quad$ heat transfer coefficient 


\begin{tabular}{|c|c|}
\hline$K_{f}$ & $\begin{array}{l}\text { thermal conductivity of air at film tem- } \\
\text { perature }\end{array}$ \\
\hline$L$ & test channel length \\
\hline$N u$ & $\begin{array}{l}\text { regionally averaged Nusselt number, } \\
h D_{H} / K_{f}\end{array}$ \\
\hline$\overline{N u}$ & $\begin{array}{l}\text { channel (first, second channel and U- } \\
\text { bend region) averaged Nusselt number }\end{array}$ \\
\hline$p$ & pitch \\
\hline $\begin{array}{l}q_{\ell}, q_{t} \\
Q_{t}\end{array}$ & $\begin{array}{l}\text { local heating boundary conditions } \\
\text { the total power input to the test section } \\
\text { midspan eccentricity }(300 \mathrm{~mm})\end{array}$ \\
\hline$R a_{\Omega}$ & $\begin{array}{l}\text { rotational Rayleigh number, } \beta \Delta T_{m} \bar{R} \\
(\operatorname{ReR} o)^{2} \operatorname{Pr} / K_{f}\end{array}$ \\
\hline$R e_{H}$ & Reynolds number, $U D_{H} / \nu$ \\
\hline $\operatorname{Re}_{\Omega}$ & rotational Reynolds number, $\Omega D_{H}^{2} / \nu$ \\
\hline Ro & rotation number, $R e_{\Omega} / R e_{H}\left(=\Omega D_{H} / U\right)$ \\
\hline$T$ & local temperature \\
\hline$T_{b i}$ & bulk temperature at inlet \\
\hline$T_{b x}$ & local bulk temperature \\
\hline$T_{f}$ & local film temperature $=\left(T_{w}+T_{b w}\right) / 2$ \\
\hline$T_{w}$ & local wall temperature \\
\hline$T_{0}$ & free stream temperature \\
\hline$U$ & $\begin{array}{l}\text { mean channel velocity/throughflow ve- } \\
\text { locity }\end{array}$ \\
\hline$W$ & width of channel \\
\hline$x$ & downstream distance \\
\hline$\beta$ & $\begin{array}{l}\text { coefficient of thermal expansion and } \\
\text { model orientation }\end{array}$ \\
\hline$\rho$ & density of air \\
\hline$\Delta P$ & pressure drop $\left(=P_{x}-P_{i}\right)$ \\
\hline$\Delta T_{m}$ & $\begin{array}{l}\text { used in calculation of } R a \text { which was } \\
\text { chosen at an average of inlet and exit } \\
\text { fluid bulk temperature differences for all } \\
\text { the rotational speeds under study }\end{array}$ \\
\hline$(\Delta \rho / \rho)_{i}$ & $\begin{array}{l}\text { density ratio based on the inlet bulk } \\
\text { temperature }\end{array}$ \\
\hline$\eta$ & $\begin{array}{l}\text { enhancement performance ratio, }(N u / \\
\left.N u_{0}\right) /\left(f \mid f_{0}\right)\end{array}$ \\
\hline & kinematic viscosity of air \\
\hline & rotational speed \\
\hline
\end{tabular}

Subscripts

0 free stream/discharge

$i \quad$ inlet

$o \quad$ smooth and stationary

$x \quad$ local position in downstream

$t \quad$ total

w wall

$\Omega \quad$ rotational

\section{References}

Han, J. C., Zhang, Y.-M. and Kalkuehler, K. (1993) Uneven Wall Temperature Effect on Local Heat Transfer in a Rotating Two-pass Square Channel with Smooth Walls, ASME Journal of Heat Transfer, 115, 912-920.

Hong, Y. J. and Hsieh, S. S. (1993) Heat Transfer and Friction Factor Measurements in Ducts with Staggered and In-line Ribs, ASME Journal of Heat Transfer, 115, 58-65.

Hsieh, S. S. and Hong, Y. J. (1995) Heat Transfer Coefficients in an Orthogonally Rotating Duct with Turbulators, ASME Journal of Heat Transfer, 117, 69-78.

Hsieh, S. S. and Liu, W. J. (1996) Uneven Wall Heat Flux Effect on Local Heat Transfer in Rotating Two-pass Channels with Two Opposite Ribbed Wall, ASME Journal of Heat Transfer, 118, 864-876.

Parsons, J. A., Han, J.-C. and Zhang, Y. M. (1994) Wall Heating Effect on Local Heat Transfer in a Rotating Twopass Square Channel with $90^{\circ}$ Rib Turbulators, Int'l. J. Heat Mass Transfer, 37, 1411-1420.

Parsons, J. A., Han, J.-C. and Zhang, Y. M. (1995) Effect of Model Orientation and Wall Heating Condition on Local Heat Transfer in a Rotating Two-pass Square Channel with Rib Turbulators, Int'l. J. Heat Mass Transfer, 38, $1151-1159$.

Prakash, C. and Zerlke, R. (1992) Prediction of Turbulent Flow and Heat Transfer in a Radially Rotating Square Duct, ASME Journal of Turbomachinery, 114, 835-846.

Rohsenow, W. M. and Choi, H. (1961) Heat, Mass and Momentum Transfer, Prentice-Hall, Englewood Cliffs, NJ, pp. $192-193$.

Wagner, J. H., Johnson, B. V., Graziani, R. A. and Yeh, F. C. (1992) Heat Transfer in Rotating Serpentine Passages with Trips Normal to the Flow, ASME Journal of Turbomachinery, 114, 847-857.

Yang, W. J., Zhang, N. and Chiou, J. (1992) Local Heat Transfer in a Rotating Serpentine Flow Passage, ASME Journal of Heat Transfer, 114, 354-361.

Zhang, Y. M., Han, J. C., Parsons, J. A. and Lee, C. P. (1995) Surface Heating Effect on Local Heat Transfer in a Rotating Two-pass Square Channel with 60 deg Angled Rib Turbulators, ASME Journal of Turbomachinery, 117, 272-280. 

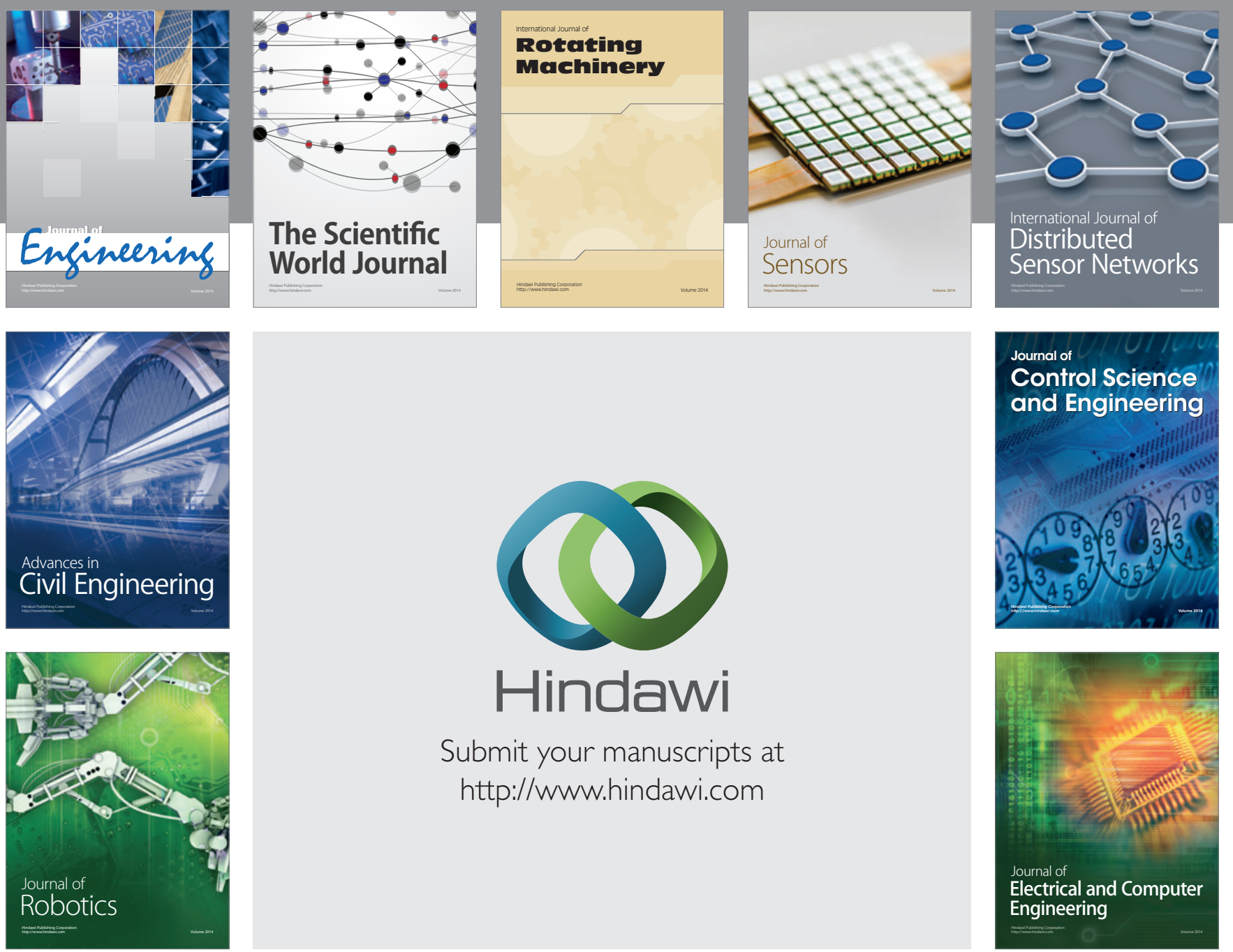

Submit your manuscripts at

http://www.hindawi.com
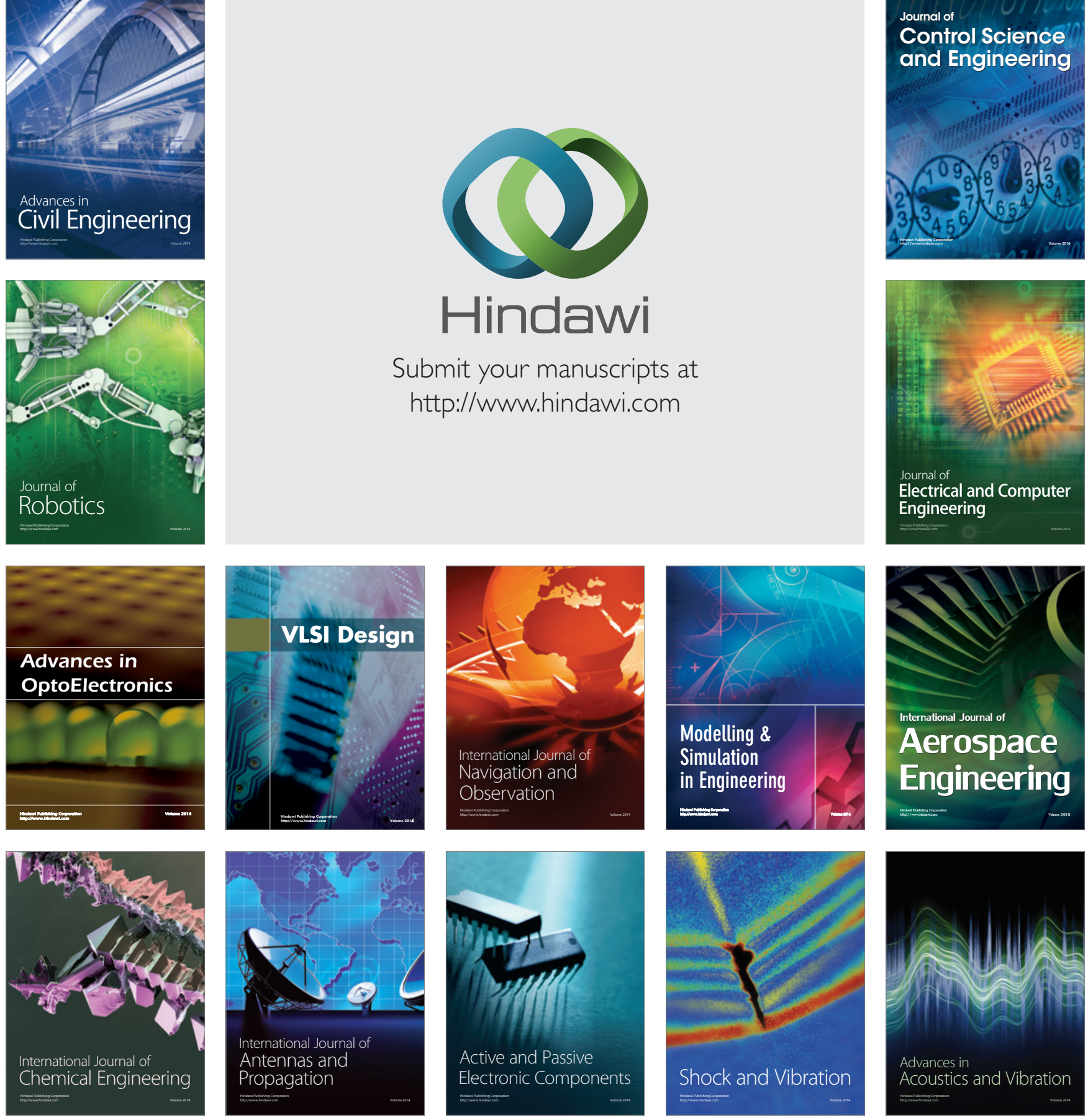